\title{
MODELIZACIÓN DE LA PÉRDIDA DE SUELO EN UNA CUENCA ENDORREICA DEL PIRINEO OSCENSE
}

\author{
M. LÓPEZ-VICENTE, A. NAVAS, J. MACHIN y L. GASPAR \\ Departamento de Suelo y Agua, \\ Estación Experimental de Aula Dei, CSIC. \\ Apartado 202, 50080 Zaragoza
}

Correo electrónico de contacto: mvicente@eead.csic.es

RESUMEN. La erosión es una seria amenaza en las regiones mediterráneas, por lo tanto la estimación y cuantificación de las pérdidas de suelo a escala de cuenca se hacen necesarias. Para promover medidas de conservación y valorar la vulnerabilidad del humedal de la cuenca del Estanque de arriba, situado en la provincia de Huesca, se ha aplicado el modelo semiempírico de erosión SEMMED. Este modelo calcula las tasas de desagregación de partículas de suelo por impacto de las gotas de lluvia, así como la de capacidad de transporte de la escorrentía, para cada píxel de la cuenca, permitiendo obtener un mapa final de pérdida de suelo anual. La integración del modelo digital de elevaciones de la zona de estudio, junto con la base de datos climática y analítica en la aplicación ArcGIS 9.0, ha permitido generar los mapas de los diferentes parámetros y factores del modelo. Para obtener los datos requeridos se llevó a cabo una campaña de campo recogiendo un total de 80 muestras de suelo. El mapa de flujo superficial acumulado ha sido calculado mediante los algoritmos de flujo sencillo, flujo múltiple y flujo combinado. El algoritmo de flujo múltiple se ha mostrado como la mejor propuesta para modelizar el patrón de flujo de la escorrentía, especialmente en las zonas de poca pendiente. La tasa media de desagregación del suelo por impacto de las gotas de lluvia y la de capacidad de transporte han sido de 11.79 y $9641.28 \mathrm{~kg} \mathrm{~m}^{-2}$, respectivamente, mientras que la tasa media de pérdida de suelo anual ha sido de $9.02 \mathrm{~kg} \mathrm{~m}^{-2}$ año-1. Los campos de cultivo de cereal y los caminos presentan las mayores tasas de pérdida de suelo, mientras que las áreas asociadas a texturas franco arenosas y con elevados valores de conductividad hidráulica saturada presentan las menores tasas de erosión. Las zonas situadas en las divisorias exterior e interiores de la cuenca y aquellas con pendiente cero, presentan un valor nulo de pérdida de suelo.

ABSTRACT. Erosion is a serious threat in Mediterranean regions, therefore assessment and quantification of soil losses at watershed scale is necessary. To 
promote soil conservation and assess the vulnerability of wetlands in the Estanque de arriba catchment, which is located in the province of Huesca (Spain), the semi-empirical soil erosion model SEMMED has been used. This model is based on the assessment of both the splash detachment and the runoff transport capacity rates per raster cell within the catchment to obtain a final map of annual soil erosion rate. Integration of the digital elevation model of the study area and the climatic and analytical database in the ArcGIS 9.0 has allowed generating maps for the different parameters and factors. In order to obtain the required values a field survey was carried out collecting a total of 80 samples of soil. The cumulative overland flow map has been calculated with the single flow, multiple flow and combined flow algorithms. The multiple flow algorithm has showed up as the best approach for modeling the drainage pattern of runoff, especially in flat areas. The mean splash detachment and transport capacity rates are 11.79 and $9641.28 \mathrm{~kg} \mathrm{~m}^{-2}$, respectively, whereas the mean rate of annual soil loss is $9.02 \mathrm{~kg} \mathrm{~m}^{-2}$ year-1. Crops of cereal and paths present the highest rates of soil loss, whereas the areas associated with sandy loam textures and high values of saturated hydrological conductivity present the lowest values. The external and internal boundaries of the catchment are characterized by no erosion processes.

Palabras clave: impacto de la lluvia, capacidad de transporte, flujo acumulado múltiple, modelo SEMMED, SIG, Pirineos, humedal, Estaña.

Key words: splash, transport capacity, multiple flow accumulation, SEMMED model, GIS, Pyrenees, wetland, Estaña.

Enviado el 16 de octubre de 2006 Aceptado el 23 de noviembre de 2006

\section{Introducción}

Los procesos de cambio global están conduciendo hacia la desertificación y regresión de los humedales en las regiones mediterráneas, perdiendo áreas de alto índice de biodiversidad. En Europa, los países del área mediterránea son los que más riesgo presentan de sufrir cambios ambientales que afectarán a los recursos de agua y suelo y que podrían conducir a la desertificación. Nearing et al. (2004) indican que el calentamiento global conducirá a un ciclo hidrológico más vigoroso con una mayor frecuencia de eventos de mayor intensidad pluviométrica.

Los procesos de erosión en la cuenca aportan sedimentos que se acumulan en las zonas deprimidas y hacen peligrar la conservación de los lagos y humedales. Los modelos de pérdida de suelo integrados en Sistemas de Información Geográfica (SIG) son herramientas útiles para la estimación de los procesos de degradación del suelo a escala de cuenca y regional.

El objetivo de este trabajo es estimar la pérdida de suelo promedio anual en una cuenca endorreica del Prepirineo oscense, asociada a una laguna de pequeñas dimensio- 
nes, conocida bajo el nombre de "Estanque de arriba" y siendo parte del paraje de los Lagos de Estaña (Riera et al., 2006). Esta cuenca presenta un fuerte contraste litológico en las formaciones superficiales y en los diversos elementos topográficos. Se pretende identificar las principales áreas con contribución neta de agua y sedimento que alcanzan la laguna y su entorno, así como analizar la importancia de los diferentes algoritmos de flujo acumulado ponderado en la estimación final de la tasa de capacidad de transporte de la escorrentía. Para alcanzar estos objetivos se ha aplicado el modelo semiempírico de erosión SEMMED (De Jong, 1994; De Jong et al., 1999), así como diversos algoritmos de ruta geomorfológicos, no incluidos en el modelo original. El resultado de este trabajo pretende aportar información detallada acerca de los actuales procesos de degradación del terreno que afectan a los humedales en agroecosistemas mediterráneos de montaña, así como ayudar en la política de conservación y recuperación de estos paisajes bajo las amenazas del actual marco de cambio climático.

\section{Material y métodos}

\section{1. Área de estudio}

La cuenca del "Estanque de arriba" se sitúa en el noreste de España, en el límite oriental de la provincia de Huesca (Fig. 1a), en el paraje conocido como Lagos de Estaña, a una altitud comprendida entre 679 y 892 m.s.n.m. Este paraje se sitúa en la parte central del Prepirineo, entre los ríos Cinca y Noguera Ribagorzana, próxima al límite norte de la cuenca del Ebro, caracterizado en los alrededores de la zona de estudio por pliegues de dirección E-O que forman parte de las llamadas Sierras Exteriores (Rodríguez-Fernández, 2004). Los núcleos de los anticlinales de estas sierras aparecen perforados por afloramientos de diapiros triásicos compuestos principalmente por margas gipsíferas, dolomías, calizas, ofitas y depósitos salinos ocasionales. Éstos últimos conducen los procesos de karstificación con el desarrollo de dolinas (Bischoff et al., 1994), algunas de las cuales alcanzan el nivel freático regional (IGME, 1982), dando lugar a la formación de lagunas. Estudios regionales realizados en las inmediaciones de los Lagos de Estaña han puesto de manifiesto su naturaleza y origen kársticos, al haber formado parte del primitivo poljé de Saganta, y estar localizado hoy en día al este del actual poljé de Saganta (Sancho Marcén, 1988) y al oeste de la Depresión de Estopiñán-Caserras (Martín-Serrano et al., 2005), desarrollada esta última sobre materiales triásicos salinos. Riera et al. (2004) presentan en una reciente publicación el mapa con los principales elementos geológicos del paraje de los Lagos de Estaña, incluyendo las dolinas asociadas con las lagunas, así como otras dolinas desarrolladas a lo largo de la ladera. Tras el análisis detallado de la topografía y del modelo digital de elevaciones (MDE) de este paraje López-Vicente y Navas (2005a) definen los límites de las cuencas occidentales de los Lagos de Estaña, calculando una extensión total de 74.5 ha para la cuenca del "Estanque de arriba", y de 1.7 ha para la laguna. Julià (1980) propone una tasa de sedimentación superior a $1 \mathrm{~mm}$ año ${ }^{-1}$ para las lagunas kársticas desarrolladas en ambientes de media montaña del Pirineo oriental. 


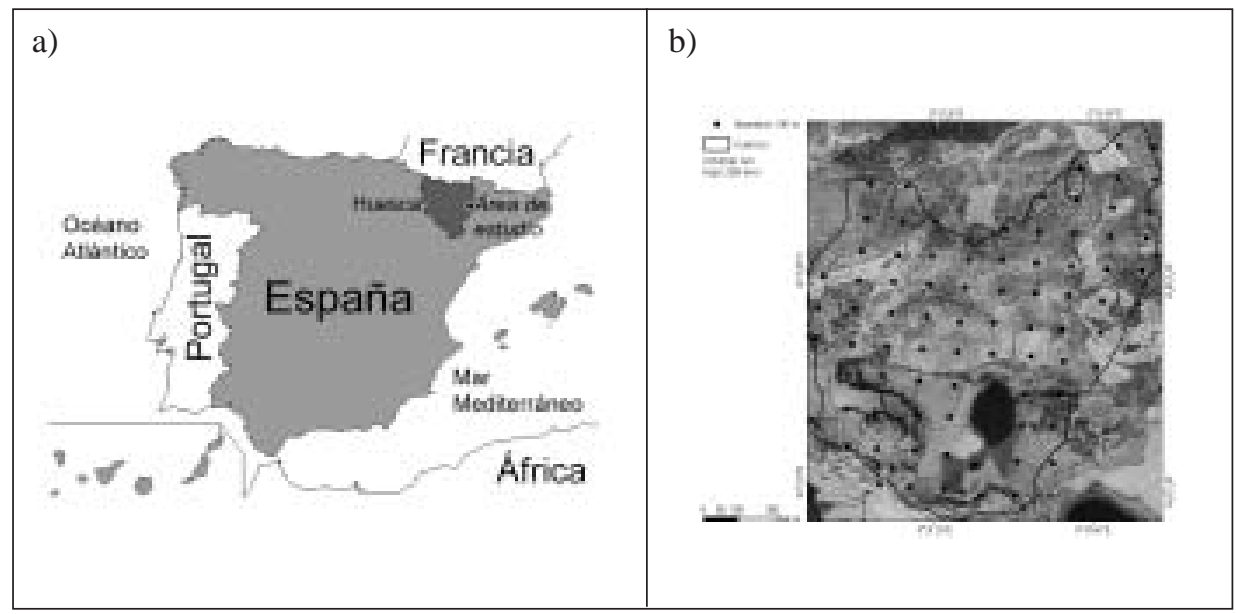

Figura 1. Situación geográfica de la zona de estudio en la provincia de Huesca (a). Límite de la cuenca del "Estanque de arriba" y localización de los puntos de muestreo sobre la ortofoto en blanco y negro de la zona de estudio (b)

Esta zona presenta un clima mediterráneo continental con dos periodos de alta intensidad de precipitación, en primavera y otoño. La precipitación media anual es de 589 mm (estimada para el periodo 1997-2005), con una oscilación interanual de $254 \mathrm{~mm}$ en 2005 y $923 \mathrm{~mm}$ en 2003. La temperatura media anual es de $12.8^{\circ} \mathrm{C}$, siendo enero el mes más frío (valor medio de $4.2^{\circ} \mathrm{C}$ ) y julio el mes más cálido (valor medio de $21.6^{\circ} \mathrm{C}$ ). López-Vicente et al. (2005b) calcularon los valores de precipitación y temperatura diaria para la zona de estudio utilizando datos de las estaciones meteorológicas de Benabarre y Camporrels para el periodo 1997-2004. Los datos correspondientes a 2005 han sido añadidos en este estudio.

\subsection{Métodos}

El modelo de erosión SEMMED (Soil Erosion Model for Mediterranean Areas) fue propuesto por De Jong et al. (1994) y aplicado en la provincia de Ardèche, al sur de Francia, en una cuenca de $12 \mathrm{~km}^{2}$ bajo un clima sub-mediterráneo con una cubierta vegetal formada por bosques de robles, matorrales y cultivos de viña y huerta. Este modelo de erosión es una versión mejorada del modelo empírico Morgan, Morgan and Finney (MMF, Morgan et al., 1984), al utilizar las ventajas derivadas de la aplicación de los SIG en el cálculo de parámetros distribuidos así como del análisis de las variables topográficas de pendiente y del cálculo del flujo acumulado ponderado. Además, incorpora un factor relativo a la conductividad hidráulica saturada que no aparece en el modelo MMF. El modelo SEMMED ha sido aplicado a escala regional en diversas cuencas mediterráneas, como son la cuenca de La Peyne, en la provincia de Hérault, al sur de Francia (De Jong et al., 1998), en la cuenca Simeto $\left(4200 \mathrm{~km}^{2}\right.$ ), al este de Sicilia (De 
Jong et al., 1999; Paracchini et al., 1997) y en la cuenca Shiqma $\left(760 \mathrm{~km}^{2}\right)$ en el centro de Israel (Cohen et al., 2005), demostrando su capacidad de producir mapas regionales de riesgo de erosión, que resultan más prácticos en la planificación del uso y gestión del suelo que las extrapolaciones a partir de datos experimentales en parcelas.

El modelo SEMMED calcula en cada píxel la tasa de desagregación de partículas de suelo por el impacto de la gota de lluvia, así como la capacidad de transporte de la escorrentía. La tasa anual de pérdida de suelo corresponde el valor mínimo de las dos tasas anteriormente calculadas, al considerar que no hay pérdida de suelo por impacto de lluvia por encima de la tasa de capacidad de transporte calculada, y viceversa. No obstante, este modelo presenta una serie de limitaciones, al no considerar la desagregación de partículas por escorrentía, el transporte por impacto de lluvia ni el efecto de las costras superficiales, relativamente frecuentes en ambientes mediterráneos.

\subsection{Desagregación del suelo por impacto de las gotas de lluvia}

La tasa de desagregación de partículas de suelo por impacto de las gotas de lluvia $\left(F, \mathrm{~kg} \mathrm{~m}^{-2}\right)$ en el modelo SEMMED se calcula a partir de la siguiente expresión:

$$
F=K(\operatorname{Eexp}(-a A))^{b} 10^{-3}
$$

donde $K\left(\mathrm{~g} \mathrm{~J}^{-1}\right)$ es el índice de desagregabilidad del suelo estimado a partir del análisis textural, $E\left(\mathrm{~J} \mathrm{~m}^{-2}\right)$ es el factor de energía de la lluvia, $A(0-1)$ es el factor de intercepción de la lluvia por la vegetación, y $a$ y $b$ son coeficientes $(\mathrm{a}=0.05 ; \mathrm{b}=1.0)$. El valor de la energía total de la lluvia se obtiene a partir de la ecuación:

$$
E=R\left(11.9+8.7 \log _{10} I\right)
$$

donde $R(\mathrm{~mm})$ es la precipitación promedio anual, e $I\left(\mathrm{~mm} \mathrm{~h}^{-1}\right)$ es la intensidad característica de la lluvia erosiva anual.

\subsection{Capacidad de transporte de la escorrentía}

La capacidad de transporte de la escorrentía $\left(G, \mathrm{~kg} \mathrm{~m}^{-2}\right)$ está en función de la capacidad de transporte distribuida, $D T$, de la pendiente de la ladera ( $S$, grados) y del factor de la cubierta vegetal $(C, 0-1)$ definido en el modelo de erosión RUSLE (Renard et al., 1997). El cálculo de la capacidad de transporte sigue la siguiente expresión:

$$
\begin{aligned}
& G=C \cdot D T^{1.7} \cdot \sin (S) \cdot 10^{-3} \\
& D T=\left(C F-K_{f s}\right) \cdot \sin (S) \cdot C H
\end{aligned}
$$

donde $C F(\mathrm{~mm})$ es el flujo superficial acumulado, $K_{f s}\left(\mathrm{~mm} \mathrm{día}^{-1}\right)$ es la conductividad hidráulica saturada calculada para cada tipo de suelo y $C H(0,1)$ es el factor de la red hidrológica, que identifica las zonas con un curso de agua permanente, descartándolas 
del cálculo final de la erosión. En este trabajo se ha asociado el valor 0 a la lagunas y 1 al resto de la cuenca, al no existir ningún curso permanente de agua, debido a las reducidas dimensiones de la cuenca.

El mapa del flujo superficial acumulado, $C F$, se obtiene al aplicar un algoritmo de flujo acumulado ponderado sobre el mapa de flujo superficial para cada píxel, $Q_{i}(\mathrm{~mm})$. En este estudio se han aplicado tres algoritmos diferentes, de flujo sencillo, de flujo múltiple y de flujo combinado, con el objetivo de analizar las ventajas e inconvenientes de cada uno de ellos en el tratamiento del MDE, así como la adecuación de los mismos a los distintos tipos de eventos de lluvia. El flujo superficial en cada píxel se calcula según la siguiente ecuación:

$$
\begin{aligned}
& Q_{i}=R \cdot \exp \left(-R_{C} / R_{0}\right) \\
& R_{C}=1000 \cdot M S \cdot B D \cdot E H D \cdot(E t / E O)_{0.5}
\end{aligned}
$$

donde $R(\mathrm{~mm})$ es la precipitación anual, $R_{0}\left(\mathrm{~mm} \mathrm{día}^{-1}\right)$ es la cantidad de lluvia promedio por día de lluvia erosiva en el año, y $R_{C}(\mathrm{~mm})$ es el valor crítico de almacenamiento de agua en el suelo. Este último factor está en función del contenido de agua en el suelo a capacidad de campo $(M S, \%)$, de la densidad aparente del suelo $\left(B D, \mathrm{~g} \mathrm{~cm}^{-3}\right)$, de la profundidad hidrológica efectiva $(E H D, \mathrm{~m})$, y del cociente entre la evapotranspiración real y potencial $\left(E_{t} / E_{0}\right)$.

\subsection{Cálculo de los parámetros requeridos}

En un trabajo previo López-Vicente et al. (2005b) calcularon el flujo acumulado ponderado para la totalidad de las cuencas del entorno de los Lagos de Estaña utilizando un algoritmo de flujo sencillo. En este trabajo se obtuvo un valor de lluvia media por día de lluvia erosiva, factor $R_{0}$ en (5), de $16.7 \mathrm{~mm} \mathrm{~h}^{-1}$, así como unos valores medios, mínimos y máximos de almacenamiento de agua en el suelo a capacidad de campo, factor $M S$ en (6), y de densidad aparente, factor $B D$ en (6), de $28.7,15.9$ y $52.0 \%$, y de 1.2 , 0.7 y $2.3 \mathrm{~g} \mathrm{~cm}^{-3}$, respectivamente.

Estos autores calcularon el mapa de profundidad hidrológica efectiva, factor $E H D$ en (6), para cada tipo de suelo, obteniendo un valor mínimo de 5 centímetros para leptosoles líticos y máximo de 25 centímetros para gleisoles y leptosoles renzicos. Además, calcularon la conductividad hidráulica saturada para cada tipo de suelo, obteniendo unos valores mínimo y máximo de 9.9 y $2252.5 \mathrm{~mm} \mathrm{día}^{-1}$, para gipsisoles háplicos y leptosoles háplicos, respectivamente. Los mapas correspondientes a los factores de profundidad hidrológica efectiva y de conductividad hidráulica saturada han sido corregidos en aquellas zonas donde aparecen canchales, debido a la no existencia de suelo, en sentido estricto, en estos puntos, y a la elevada conductividad hidráulica de los mismos, modificando ambos factores de manera notable, la dinámica superficial de la escorrentía, así como la tasa final de erosión del suelo.

El cociente entre la evapotranspiración real y potencial para cada tipo de cubierta vegetal, así como el factor de cubierta de vegetación, factor $C$ en (3), fueron calculado por López-Vicente et al. (2005b), mediante el simulador de sistemas de cultivo CropSyst 3.05. 
Los datos necesarios para calcular el valor de intensidad característica de la lluvia erosiva anual, $I$ en (2), han sido obtenidos de la estación meteorológica de Canelles, situada a 11 kilómetros al sureste de la zona de estudio. La serie temporal disponible abarca de 1997 a 2005 con datos de precipitación acumulada cada 15 minutos. Se ha seleccionado esta estación al ser la más próxima a la zona de estudio con esta resolución temporal. Los eventos de lluvia erosiva fueron diferenciados del total de eventos de lluvia siguiendo los criterios de Renard et al (1997) expuestos la guía del modelo de erosión RUSLE. El factor de desagregabilidad del suelo, $K$ en (1), fue calculado a partir de la clasificación texturales de 80 muestras de suelo recogidas según una malla regular de 100 metros, en la cuenca del "Estanque de arriba" (Fig. 1b). El análisis textural se llevó a cabo con un equipo de medida láser.

Belmonte y Romero (1998) estimaron la intercepción neta de la lluvia por la vegetación, $A$ en (1), en un 33.8 y $30.8 \%$ para el bosque mediterráneo abierto y para el matorral, respectivamente, en el sureste de España. Ashby (1999) calculó la tasa de intercepción de la lluvia para la cubierta de pastos en un $13.1 \%$. En este artículo no se han considerado las variaciones mensuales del parámetro de intercepción en los campos de cultivo, considerando que el suelo asociado con esta cubierta vegetal permanece desnudo durante todo el año. Este criterio ha sido adoptado tras analizar las variaciones mensuales del factor de intensidad de la lluvia, observando una relación directa entre los meses con los valores más altos de intensidad y aquellos en los que el suelo de los campos de cultivo permanecen desnudos tras la cosecha, y por lo tanto, la posible cubierta vegetal remanente no realiza un verdadero papel de intercepción de la lluvia.

El MDE de la zona de estudio, corregido por López-Vicente y Navas (2005a), presenta una resolución espacial de 5 x 5 metros y ha sido la base para el cálculo del mapa de pendiente, factor $S$ en (3) y (4). El cálculo del flujo acumulado ponderado, factor $C F$ en (4), se ha llevado a cabo con tres algoritmos diferentes, que son el de flujo sencillo (D8), el de flujo múltiple (MD) y el de flujo combinado (MDD8), incluidos en la extensión HydroTools 1.0 para ArcView3.x. Esta extensión ha sido desarrollada por Holger Schäuble, siendo de libre acceso a través de internet (www.terracs.de/Overview/overview.html).

Finalmente, todos los datos climáticos, de medidas de campo y de laboratorio, así como el MDE y los algoritmos de ruta fueron integrados en el programa de tipo SIG ArcGIS 9.0, que permitió la elaboración de los mapas requeridos, así como el cálculo de las tasas de desagregación de partículas de suelo y de capacidad de transporte según las ecuaciones del modelo SEMMED.

\section{Resultados y discusión}

Tras analizar un total de 606 eventos de lluvia registrados en la estación meteorológica de Canelles, para el periodo 1997-2005, se distinguieron 114 eventos de lluvia erosiva, con un valor promedio de intensidad característica de la lluvia erosiva, factor $I$ en (2), de $16.7 \mathrm{~mm} \mathrm{~h}^{-1}$. La energía cinética total de la lluvia fue calculada siguiendo la 
ecuación (2), obteniendo un valor final de $13696.01 \mathrm{~J} \mathrm{~m}^{-2}$. Tras analizar la textura de las 80 muestras de suelo recogidas y siguiendo los valores propuestos por Morgan et al (1984) se calculó el índice de desagregabilidad del suelo para cada muestra, factor $K$ en (1). El método de interpolación spline, incluido en ArcGIS 9.0, fue utilizado para generar el mapa correspondiente al factor $K$ (Fig. 2a).

A partir del mapa de cubiertas de vegetación elaborado por López-Vicente et al. (2005b) para el conjunto del paraje de los Lagos de Estaña, y de los valores propuestos por Belmonte y Romero (1998) y Ashby (1999) se elaboró el mapa del factor A de intercepción de la lluvia por la vegetación (Fig. 2b). Las cubiertas correspondientes a caminos, suelos desnudos, canchales y campos de cultivo de cebada presentan un valor cero de intercepción neta, mientras que la vegetación de ribera que rodea la laguna así como los píxeles asociados con edificios y caminos asfaltados, presentan un valor máximo de 100, asociado con una intercepción total de la lluvia. Este último mapa, junto con los de desagregabilidad del suelo y de energía cinética, han permitido calcular y generar el mapa de desagregación del suelo por impacto de las gotas de lluvia según la ecuación (1) (Fig. 2c), obteniendo un valor

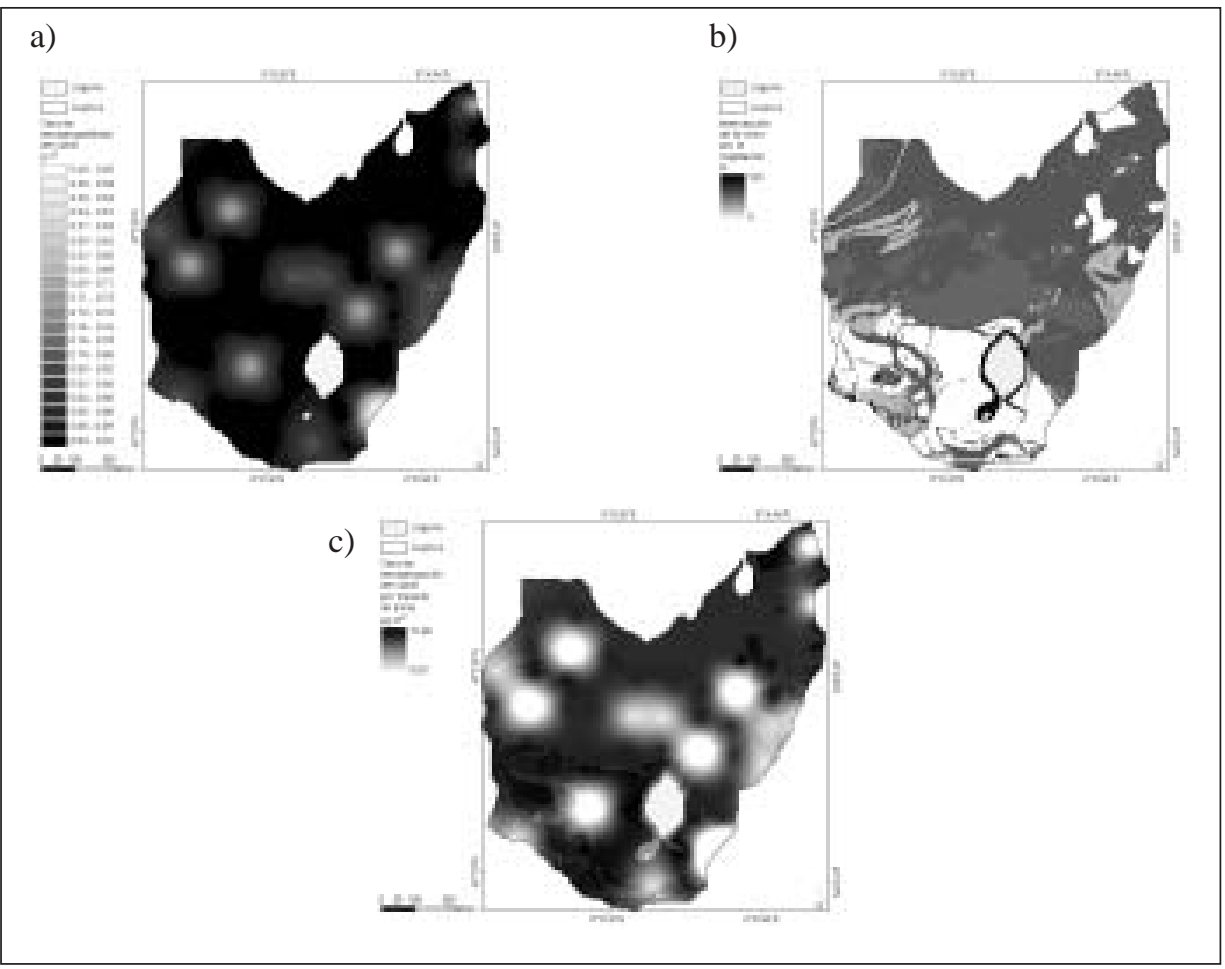

Figura 2. Mapa del factor $K$ de desagregabilidad del suelo (a). Mapa del factor $A$ de intercepción de la lluvia por la vegetación (b). Mapa de la tasa de desagregación del suelo por impacto de la lluvia, $F(c)$ 
medio de $11.79 \mathrm{~kg} \mathrm{~m}^{-2}$ de pérdida potencial de suelo. Este mapa pone de manifiesto el fuerte control del factor $K$ de desagregabilidad del suelo sobre el resultado final obtenido, correspondiendo los valores de mínima erosión potencial con aquellos puntos asociados a muestras de textura franco-arenosa, en comparación con el resto de las muestras, de textura franco-limosa. Los valores mínimo y máximo obtenidos son de 5.53 y $12.48 \mathrm{~kg} \mathrm{~m}^{-2}$, respectivamente, asociándose los valores más altos con las áreas limítrofes a la laguna.

El mapa correspondiente al valor crítico de almacenamiento de agua en el suelo, $R_{C}$, fue calculado siguiendo la ecuación (6) obteniendo un valor medio de $22.51 \mathrm{~mm}$ por píxel, y un valor mínimo y máximo de 0 y 75.08 mm, respectivamente (Fig. 3a). El mínimo valor obtenido corresponde a aquellas zonas con presencia de canchales $(<1 \%$ del área total), mientras que los valores más altos corresponden a los suelos con mayor profundidad hidrológica efectiva. Este último factor aparece como el más importante en el cálculo del almacenamiento de agua en el suelo, mientras que los factores de evapotranspiración y de densidad aparente, son los menos influyentes. El factor del contenido en agua del suelo a capacidad de campo ejerce un papel intermedio.

A partir del mapa de almacenamiento de agua en el suelo y de los parámetros de lluvia calculados por López-Vicente et al (2005b), se obtuvo el mapa del parámetro $Q_{i}$ del flujo superficial por píxel (Fig. 3b). Los valores medio, mínimo y máximo obtenidos son de 262.01, 61.37 y $468.07 \mathrm{~mm}$, respectivamente.

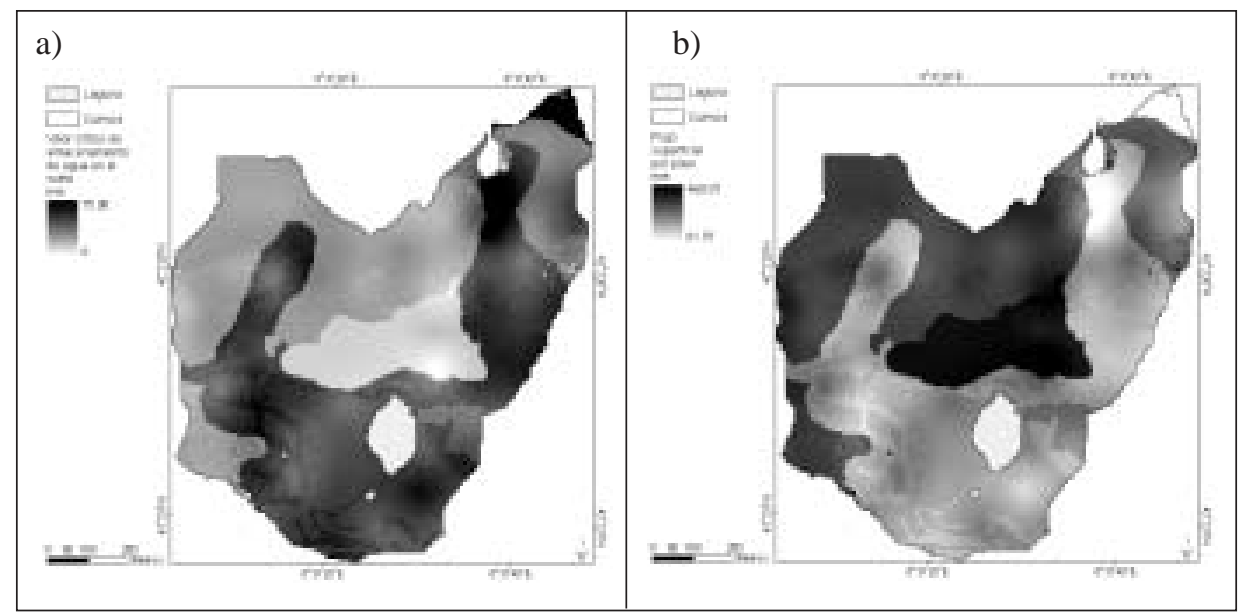

Figura 3. Mapa del factor $R_{C}$ de almacenamiento de agua en el suelo (a). Mapa del factor $Q_{i}$ de flujo superficial por píxel (b)

Tras aplicar el algoritmo de flujo sencillo ponderado sobre el mapa de flujo superficial se obtuvo el mapa del factor $C F$ de flujo superficial acumulado correspondiente a una red de drenaje dendrítica. El valor máximo obtenido ha sido de 2739088 mm (Fig. 
4a) mientras que el máximo valor del factor $C F$ obtenido tras aplicar el algoritmo de flujo múltiple ponderado, asociado con un patrón de flujo laminar, ha sido de 1371467 mm (Fig. 4b), que es menos de la mitad del valor obtenido tras aplicar el algoritmo de flujo sencillo. Finalmente, se aplicó el algoritmo de flujo combinado, obteniendo un valor máximo de $2720070 \mathrm{~mm}$ (Fig. 4c), muy próximo al valor obtenido con el algoritmo de flujo sencillo. Se ha seleccionado un valor umbral de 20000 entre el algoritmo de flujo múltiple y el de flujo sencillo para la aplicación del algoritmo combinado. Este valor ha sido propuesto tras analizar la ortofoto de la zona de campo y localizar la situación geográfica del inicio de los gullies en cabecera, haciendo coincidir este punto con el cambio de flujo laminar a flujo sencillo.

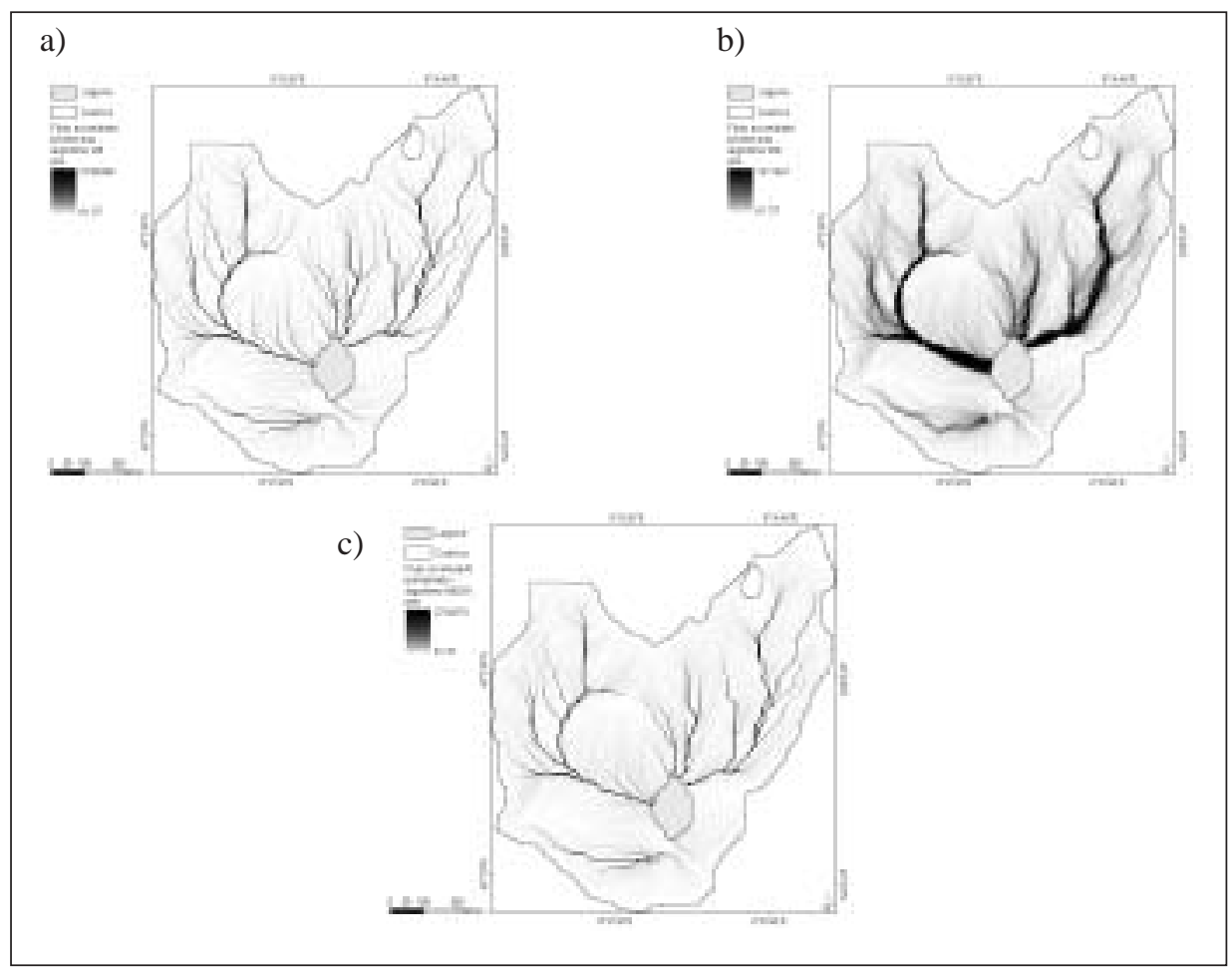

Figura 4. Mapa del factor CF de flujo superficial acumulado calculado con el algoritmo de flujo sencillo D8 (a). Mapa del factor CF calculado con el algoritmo de flujo múltiple MD

(b). Mapa del factor CF calculado con el algoritmo de flujo combinado MDD8 (c)

El mapa del factor $C F$ seleccionado para el cálculo de la capacidad de transporte distribuida, factor $D T$ en (4), ha sido el calculado con el algoritmo de flujo múltiple, al considerar que representa el patrón de escorrentía más próximo a la realidad para la mayor parte de los eventos de lluvia. No obstante, en aquellos eventos de lluvia extrema, con 
picos de intensidad muy alta, el mapa del factor $C F$ deberá ser calculado con el algoritmo de flujo combinado, al representar con mayor exactitud la formación de cursos efímeros de agua superficial. No obstante, este último algoritmo incluye un error al alcanzar las zonas de poca pendiente o planas, ya que el flujo permanece linear, cuando el realidad, se divide en varios cursos de agua, dibujando un patrón de tipo anastomosado.

El mapa de capacidad de transporte distribuida fue calculado a partir de los mapas de flujo superficial acumulado, de conductividad hidráulica saturada, de pendiente (Fig. 5a) y del factor $\mathrm{CH}$ de la red hidrológica, siguiendo la ecuación (4). El mapa resultante se integró en la ecuación (3), juntos con los de pendiente y del factor $C$ de cubierta vegetal, para obtener el mapa de capacidad de transporte de la escorrentía, factor $G$, que aportó un valor medio y máximo de 9641.28 y $5540825 \mathrm{~kg} \mathrm{~m}^{-2}$, respectivamente (Fig. 5b). Las zonas con máxima capacidad de transporte aparecen asociadas con las 3 principales redes de drenaje, que se sitúan en la mitad norte de la cuenca, dibujando el cauce de los dos barrancos situados en los extremos noreste y noroeste. El factor topográfico de pendiente ejerce un fuerte control en el resultado final obtenido, al disminuir la capacidad de transporte de la escorrentía en las zonas de reducida pendiente situadas al oeste y al sur de la laguna, así como al asignar un valor nulo de capacidad de transporte a aquellas zonas planas (4.82 \% de la superficie de la cuenca), situadas tanto en los límites de la cuenca, como en las divisorias internas de la misma. Las superficies con bajos valores de conductividad hidráulica saturada definen áreas con una contribución positiva a la capacidad de transporte mientras que los regosoles y leposoles presentan bajas tasas del factor $G$.

Finalmente, los mapas correspondientes a la tasa de desagregación de partículas por impacto de las gotas de lluvia $(F)$ y de capacidad de transporte $(G)$ fueron comparados, siguiendo las especificaciones del modelo SEMMED, para obtener el mapa de erosión de

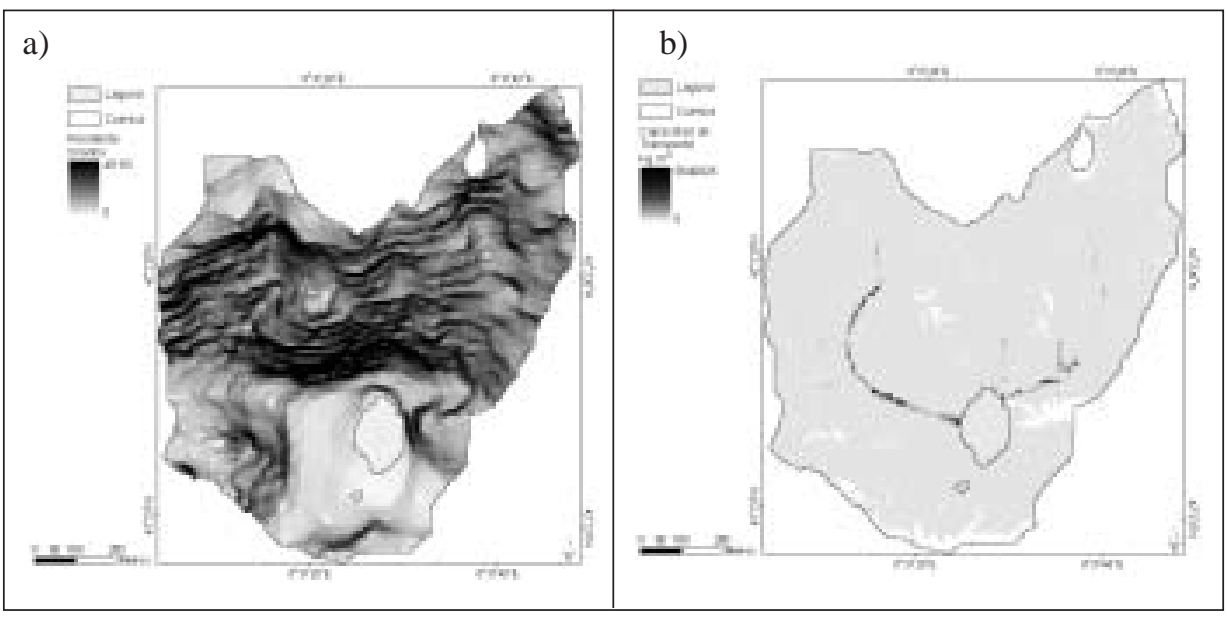

Figura 5. Mapa de pendiente de la zona de estudio (a). Mapa del factor $G$ de capacidad de transporte de la escorrentía (b) 
la cuenca (Fig. 6), que aportó un valor medio y máximo de 9.02 y $12.48 \mathrm{~kg} \mathrm{~m}^{-2}$ año $^{-1}$ de pérdida de suelo, respectivamente. Tal y como sucede en el mapa del factor $G$, los límites de la cuenca, así como las divisorias internas planas, presentan un valor nulo de pérdida de suelo. Asimismo, las áreas correspondientes con las muestras de textura franco-arenosa ofrecen una tasa de erosión significativamente inferior a la del resto de la cuenca. Las áreas cubiertas por la vegetación de ribera circundante a la laguna también presentan un valor bajo de tasa de erosión. Los máximos valores de pérdida de suelo aparecen asociados con los campos de cultivo, tanto los que rodean la laguna al oeste (valor máximo de pérdida de suelo) y al sur de la misma, como los situados en el extremo norte de la cuenca y a mitad de la ladera norte. También cabe destacar la elevada tasa de erosión calculada para el camino que cruza la cuenca en el extremo noroeste de la misma.

En el $65.58 \%$ de la cuenca, la tasa de desagregación del suelo por impacto es el factor limitante de la erosión, mientras que en el resto de la misma es la capacidad de transporte la que supone un factor limitante.

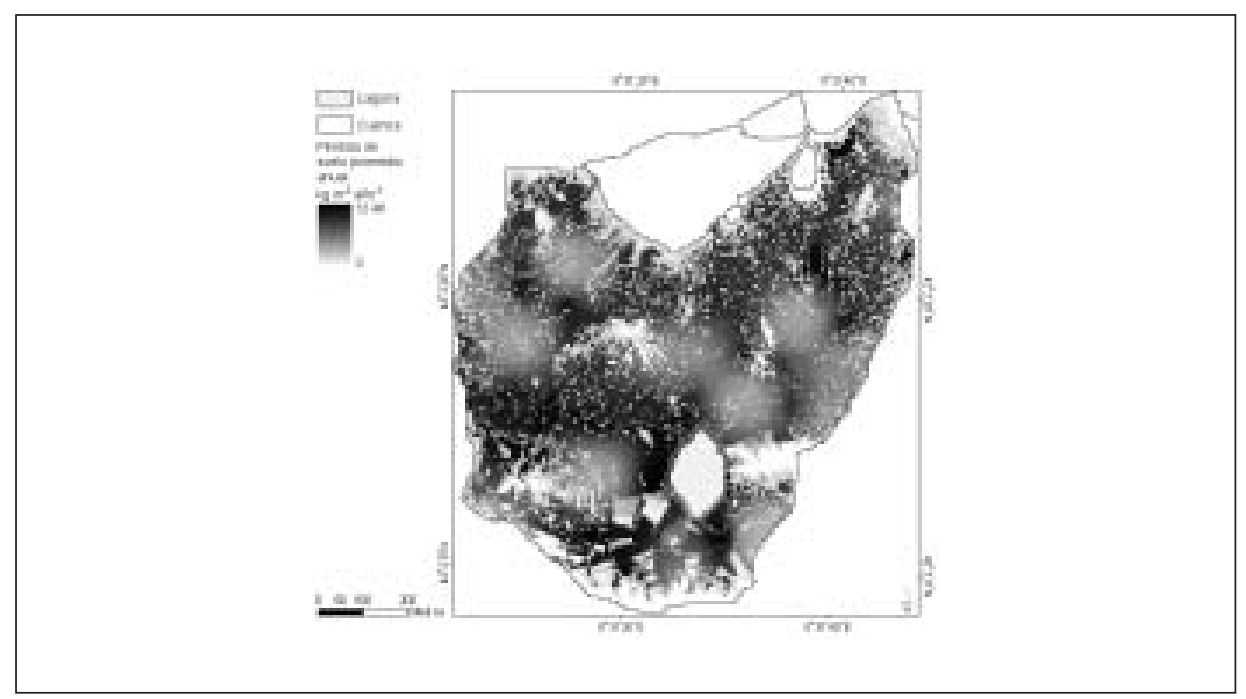

Figura 6. Mapa de la pérdida de suelo promedio anual para la cuenca del "Estanque de arriba”, calculado según el modelo de erosión SEMMED

\section{Conclusiones}

El modelo de erosión SEMMED ha permitido calcular una tasa de pérdida de suelo promedio de $9.02 \mathrm{~kg} \mathrm{~m}^{-2}$ año ${ }^{-1}$ para el conjunto de la cuenca del "Estanque de arriba". Los campos de cultivo, y los caminos aparecen como las principales fuentes de sedimento que contribuyen positivamente a la regresión del humedal y la consecuente reduc- 
ción en la extensión de la laguna, mientras que los límites de la cuenca y las divisorias internas presentan una tasa nula de pérdida de suelo. Además, los suelos asociados con textura franco-arenosa y de elevada conductividad hidráulica saturada ofrecen valores de pérdida de suelo por debajo de la media.

Aunque es necesaria una verificación de los resultados obtenidos mediante otras técnicas de estimación de la pérdida de suelo, este trabajo pone de manifiesto la importancia del uso de los SIG en el aplicación de un modelo de erosión de parámetros distribuidos, así como del análisis topográfico del MDE mediante diferentes algoritmos de flujo acumulado para la predicción de la pérdida de suelo y la identificación, tanto de las áreas estables como de aquellas que son la principal fuente de sedimentos al entorno de un humedal en un agroecosistema mediterráneo de montaña.

\section{Agradecimientos}

Esta investigación ha sido financiada por los proyectos del CICYT: REN200202702/GLO y CGL2005-02009/BTE.

\section{Referencias bibliográficas}

ASHBY, M. (1999). Modelling the water and energy balances of Amazonian rainforest and pasture using Anglo-Brazilian Amazonian climate observation study area. Agricultural and Forest Meteorology, 94: 79-101.

Belmonte, F. y Romero, A. (1998). La cubierta vegetal en las regiones áridas y semiáridas: consecuencias de la interceptación de la lluvia en la protección del suelo y los recursos hídricos, NORBA-Journal of Geography, 10: electronic journal (http://www.fylunex.com/foro/publicaciones/norba/htm_esp/Index.html).

BischOFF, J.L., JULiÀ, R., SHANKS, W.S. y RoSENBAUER, R. (1994). Karstification without carbonic acid: bedrock dissolution by gypsum-driven dedolomitization. Geology, 22: 995-998.

Cohen, S., Svoray, T. y LARonne, J.B. (2005). Catchment scale soil erosion modeling using GIS and soft computing techniques. Geophysical Research Abstracts, 7: 676.

DE Jong, S.M. (1994). Applications of reflective remote sensing for land degradation studies in a Mediterranean environment. Netherlands Geographical Studies, 177, KNAG Utrecht.

De Jong, S.M., Brouwer, L.C. y Riezebos, H. (1998). Erosion hazard assessment in the Peyne catchment, France. En: Working paper DeMon-2 Project. Dept. Physical Geography, Utrecht University. 
De Jong, S.M., Parachinni, M.L., Bertolo, F., Folving, S., Megier, J. y De Roo, A.P.J. (1999). Regional assessment of soil erosion using the distributed model SEMMED and remotely sensed data. Catena, 37(3-4): 291-308.

IGME (1982). Mapa Geológico de España 1:50.000. No. 289: Benabarre. Instituto Geológico y Minero de España, Madrid.

JuliÀ, R. (1980). La conca lacustre de Banyoles-Besalú. En: Monografies del Centre d'Estudis Comarcals de Banyoles, Banyoles.

LÓPEZ-ViCEnTE, M. y NAVAS, A. (2005a). Solving topography of Digital Elevation Model in karstic environments: a case study in the External Ranges of the Pyrenees. En: Sixth International Conference on Geomorphology (Gutiérrez, F., Gutiérrez, M., Desir, G., Guerrero, J., Lucha, P., Marín, C., García-Ruiz, J.M., Eds.). pp. 388, Zaragoza.

LóPez-Vicente, M., Nelson, R., Stockle, C.O., Navas, A. y Machín, J. (2005b). Modelización de la capacidad de transporte distribuida en subcuencas endorreicas del Pirineo oscense. En: II Simposio Nacional Sobre Control de la Degradación de Suelos (Jiménez Ballesta, R., Álvarez González, A.M., Eds.). pp. 813-817, Madrid.

Martín-Serrano, A., Nozal, F., Salazar, A. y SuÁrez, A. (2005). Geomorfología subaérea. Explicación de los elementos representados. En: Mapa Geomorfológico de España y del margen continental a escala 1:1.000.000 (Martín-Serrano, A., Ed.). pp. 23-44, IGME, Madrid.

Morgan, R.P.C., Morgan, D.D.V. y Finney, H.J. (1984). A predictive model for the assessment of soil erosion risk, J. Agric. Eng. Res., 30: 245-253.

NeARING, M.A., Pruski, F.F. y O’NeAL, M.R. (2004). Expected climate change impacts on soil erosion rates: a review. Journal of Soil and Water Conservation, 59: 43-50.

Parachinni, M.L., Minacapilli, M., Bertolo, F. y Folving, S. (1997). Soil erosion modelling and coastal dynamics: a case study from Sicily. En: Remote Sensing Society: Observations and Interactions. pp. 334-339, Nottingham.

Renard, K.G., Foster, G.R., Weesies, G.A., McCool, D.K. y Poder, D.C. (1997). Predicting Soil Erosion by Water: A Guide to Conservation Planning with the Revised Universal Soil Loss Equation (RUSLE). Handbook \#703. US Department of Agriculture, Washington, DC.

RIERA, S., LóPEZ-SÁEZ, J.A. y JuLiÀ, R. (2006). Lake responses to historical land use changes in northern Spain: The contribution of non-pollen palynomorphs in a multiproxy study, Review of Palaeobotany and Palynology, 141: 127-137.

RIERA, S., WANSARD, G. y JULÍA, R. (2004). 2000-year environmental history of a karstic lake in the Mediterranean Pre-Pyrenees: the Estanya lakes (Spain), Catena, 55(3): 293-324.

RodríGUEZ-FERnÁndeZ, L.R. (2004). Mapa Tectónico de España a escala 1:2.000.000. En: Geología de España (Vera, J.A., Ed.). SGE-IGME, Madrid.

SAncho Marcen, C. (1988). El poljé de Saganta (Sierras Exteriores Pirenáicas, prov. de Huesca). Cuaternario y Geomorfología, 2 (1-4): 107-113. 